\title{
LncRNA FTX Regulates Angiogenesis Through miR-342-3p/SPII Axis in Stroke
}

\section{Qi Gao \\ Yanfeng Wang}

Department of Neurology, The Affiliated Huaian No. I People's Hospital of Nanjing Medical University, Huai'an, 223300, Jiangsu, People's Republic of China
Correspondence: Yanfeng Wang Department of Neurology, The Affiliated Huaian No. I People's Hospital of Nanjing Medical University, Huai'an, 223300 jiangsu, People's Republic of China Email yfwang_ha@163.com
Background: Long non-coding RNAs (lnc-RNAs) and microRNAs (miRNAs) play key roles in the development of stroke. However, the role of lncRNA FTX in stroke is limited known.

Methods: Real-time polymerase chain reaction (real-time PCR) assays were used to measure the expression of lncRNA FTX, miR-342-3p and SPI1. Western blot assays were employed to examine SPI1 protein expression. The cell viability was measured by CCk8 assay. Cell migration was detected by wound healing assays and transwell assays. Angiogenesis was evaluated by matrigel tube formation assays. The interaction between lncRNA FTX, miR-342-3p and SPI1 was confirmed by site-directed mutagenesis and luciferase assays.

Results: The expression of lncRNA FTX was down-regulated in blood sample from stroke patients, MAO mice tissues and OGD/R treated BMECs. Overexpression of lncRNA FTX could increase the cell viability, migration and angiogenesis in $\mathrm{OGD} / \mathrm{R}$ treated BMECs. LncRNA FTX could act as a ceRNA for miR-342-3p. Furthermore, miR-342-3p inhibition increased migration and angiogenesis in OGD/R-induced BMECs. Dual-luciferase reporter assay verified that SPI1 was a target of miR-342-3p.

Conclusion: In summary, lncRNA FTX enhanced the angiogenesis in stroke by acting as a sponge of miR-342-3p to regulate the expression of SPI1 level.

Keywords: lncRNA FTX, angiogenesis, miR-342-3p, SPI1, stroke

\section{Introduction}

Stroke is becoming a heavy burden all over the world, especially in China, ${ }^{1}$ which occurs at a high rate, resulting in neurological dysfunction. ${ }^{2}$ Stroke was divided into two types, hemorrhagic and ischemic strokes. ${ }^{3}$ It has been reported that ischemic stroke (IS) constitutes about $85 \%$ of all stroke patients. ${ }^{4}$ Even though many efforts are being put into developing new strategies to treat ischemic stroke, the only effective treatment for stroke is intravascular thrombolysis or thrombectomy. ${ }^{5}$ Thus, to identify novel therapies seems curial, and further to reveal the mechanism of the pathological progress in stroke would provide efforts to develop the new treatment.

Angiogenesis is a process in which new blood vessels are formed by the extension or elaboration of existing vessels under physiological or pathological condition. It is known as sprouting of endothelial cells (ECs) inducing neovascularization formation under the control of an extensive variety of angiogenic stimulators and inhibitors. ${ }^{6}$ Within the process of sprouting, ECs migrate into their adjacent tissues, and then, they proliferate to form tubes to procedure novel blood vessels. $^{7}$ Under physiological conditions, angiogenesis achieves a balance between 
the encouraging and obstructing factors. When the imbalance occurs, insufficient angiogenesis would result in slow wound healing. ${ }^{8}$ When the cerebral stenosis or occlusion occurs, blood perfusion in the cerebrovascular region decreases sharply, causing a sudden drop of oxygen and nutrients and eventually leading to tissue cell damage and even death. ${ }^{9}$ Recent accumulative evidence from experimental and clinical research has established a correspondence between angiogenesis and improved neurological recovery after stroke. ${ }^{10,11}$ Nevertheless, ischemic stroke injury stimulates angiogenesis in the ischemic periphery triggered by stroke, which is inadequate to fulfill the demand for blood and removal of necrotic debris. ${ }^{12}$ Therefore, innovative and effective therapies should be developed to enhance angiogenesis for ischemic stroke treatment.

Non-coding RNAs (ncRNAs), which have been corroborated as key mediators of gene expression, include microRNAs (miRNAs), circRNAs and long ncRNAs (lncRNAs). ${ }^{13,14}$ LncRNAs are a critical kind of noncoding RNAs, which can interact with miRNAs and regulate miRNA target protein expression. ${ }^{15}$ Recently, emerging evidence suggests that abnormal expression of lncRNAs is indicated to be associated with stroke. ${ }^{16}$ Antisense noncoding RNA in the INK4 locus (ANRIL) was upregulated and gave rise to angiogenesis in rat cerebral infarction. Mechanistically, IncRNA ANRIL could increase VEGF expression and activate the NF- $\mathrm{\kappa B}$ pathway. ${ }^{17}$ Likewise, small nucleolar RNA host gene (SNHG) expression was considerably enhanced in middle cerebral artery occlusion (MCAO) mice and OGD-treated brain microvascular endothelial cells (BMECs), bringing about caspase 3 activity augmentation and subsequent apoptosis. On top of that, SNHG could make BMEC survive through HIF1 $\alpha /$ VEGF signaling. ${ }^{18}$ A protective role involved in regulating vascular function was lncRNA-myocardial infarction-associated transcript (MIAT), whose expression was markedly amplified in endothelial cells by hypoxic or oxidative stress. ${ }^{19}$ In other words, mitigating the injury of ischemia by angiogenesis can help patients with ischemic stroke carry a better prognosis. In our study, we aimed to scrutinize the impact of lncRNA five prime to Xist (FTX) on the apoptosis, migration, and angiogenesis of OGD-treated BMECs. LncRNA FTX has been reported to be related to many cancers and has promoted the proliferation of cancer cells. ${ }^{20}$ LncRNA FTX has known functions related to vasculogenic mimicry formation and tumorigenesis in osteosarcoma. ${ }^{21}$ However, the function and mechanism of lncRNA FTX in stroke remains unclear. In addition, we also uncovered exact molecular mechanisms underlying the effects of lncRNA FTX on cell viability, cell migration, and angiogenesis of OGDtreated BMECs.

\section{Methods and Materials \\ Clinical Experiments and Grouping}

A total of 30 paired peripheral blood samples were obtained from patients with stroke and healthy volunteers at the Affiliated Huaian No.1 People's Hospital of Nanjing Medical University. Patients were excluded if any other central nervous system disease was identified. Venous blood samples $(5 \mathrm{~mL})$ were drawn from all participants. The serum was isolated by centrifuging at $1000 \times \mathrm{g}$ at $4{ }^{\circ} \mathrm{C}$ for $5 \mathrm{~min}$ and stored in liquid nitrogen. The study received approval from the Affiliated Huaian No.1 People's Hospital of Nanjing Medical University (YX-2021-071$01)$. Written consent forms were obtained from all participants before sample collection. The inclusion criteria for patients with ischemic stroke (IS) were as follows: (1) patients were aged 50 and over; (2) IS was confirmed by brain imaging (CT/MRI); (3) large artery occlusion occurred in the anterior circulation; (4) patients did not receive recanalization therapy; (5) patients without history of cardiovascular or cerebrovascular diseases; and (6) patients without history of severe lung, liver, kidney or other diseases. Clinicopathological features of the studied subjects are shown in Table 1.

\section{Cell Culture}

Primary brain microvascular endothelial cells (BMECs) were cultured in endothelial cells medium (ECM) (Sciencell, USA). 293T cells were cultured in Dulbecco's modified Eagle's medium (DMEM; Invitrogen, USA) supplemented with $10 \%(\mathrm{v} / \mathrm{v})$ fetal bovine serum (FBS; Gibco, USA). Cultured cells were maintained at $37^{\circ} \mathrm{C}$ in a humidified incubator containing $5 \% \mathrm{CO} 2$.

Table I Clinicopathological Features of Studied Subjects $(n=30)$

\begin{tabular}{|l|c|c|}
\hline Characteristics & $\begin{array}{c}\text { Healthy Controls } \\
(\mathbf{n}=15)\end{array}$ & $\begin{array}{c}\text { IS Patients } \\
(\mathbf{n}=\mathbf{1 5})\end{array}$ \\
\hline Age (years, mean \pm SD) & $60.84 \pm 3.25$ & $63.14 \pm 5.10$ \\
Gender (male, \%) & $8(53.33 \%)$ & $7(46.67 \%)$ \\
Hypertension $(\mathrm{n}, \%)$ & $1(6.67 \%)$ & $10(66.67 \%)$ \\
Diabetes mellitus $(\mathrm{n}, \%)$ & $2(13.33 \%)$ & $3(20.00 \%)$ \\
Hyperlipidemia $(\mathrm{n}, \%)$ & $5(33.33 \%)$ & $6(40.00 \%)$ \\
Homocysteine $(\mu$ mol/l, mean \pm SD) & $14.35 \pm 3.21$ & $15.36 \pm 2.74$ \\
\hline
\end{tabular}




\section{OGD/R Model}

The OGD/R model was performed to mimic ischemia in vitro. Briefly, the culture medium was removed and cells were washed with PBS for three times. Cells were placed into a hypoxic chamber $\left(95 \% \mathrm{~N}_{2}\right.$ and $\left.5 \% \mathrm{CO}_{2}\right)$ at $37^{\circ} \mathrm{C}$ with serum/glucose-free DMEM. Cells in the control group were cultured under normoxic conditions at the same time. All cells were subsequently re-perfused for $12 \mathrm{~h}$ under normal culture conditions at $37^{\circ} \mathrm{C}\left(95 \% \mathrm{O}_{2}\right.$ and $\left.5 \% \mathrm{CO}_{2}\right)$.

\section{Quantitative Real-Time PCR (qRT-PCR)}

The expression level of miR-342-3p, IncRNA FTX was detected by using qRT-PCR according to the manufacturer's protocol. Total RNA was extracted using TRIzol reagent (Invitrogen, USA) and cDNA synthesis was performed with a reverse transcription kit (Promega, USA). All reactions were run in triplicate, and the fold change was equivalent to the relative expression normalized to endogenous control (2 $-\Delta \Delta \mathrm{Ct})$.

\section{Transfection and Grouping}

Before OGD/R, BMECs cells were transfected with miR342-3p mimic, miR-342-3p inhibitor, shR-lncRNA FTX or IncRNA FTX plasmid by Lipo 3000 Transfection Reagent (Invitrogen, USA) according to the manufacturer's instructions. After 4 hours, the medium was changed by fresh DMEM.

\section{Dual Luciferase Reporter Assay}

The luciferase reporter vectors (lncRNA FTX-wt and SPI1-wt) (Genewiz, China) were created. Corresponding mutants (lncRNA FTX-mut and SPI1-mut) (Genewiz, China) were constructed by gene alteration of the supposed binding sites. Cells were each seeded with a proper density of 12 -well plates. Then, all plasmids were transfected into the prepared cells for $24 \mathrm{~h}$. After post-transfection, cells were harvested, and the firefly luciferase activity was measured using a dualluciferase reporter assay system (Promega, USA). The firefly luciferase activity was normalized by renilla luciferase activity.

\section{Cell Proliferation Assay}

The cell proliferative activity was measured by using the Cell Counting Kit-8 (CCK-8) assay (Beyotime, China). All treated cells were seeded into 96-well plates overnight at a density of $10^{4}$ cells per well. After being starved in a serum-free medium for another $24 \mathrm{~h}$, cells were incubated with CCK-8 reagent. The value of absorbance was detected at $450 \mathrm{~nm}$.

\section{Transwell Assay}

After transfection, BMECs $\left(5 \times 10^{4}\right)$ in $100 \mu \mathrm{L}$ of serumfree medium were seeded into 24-well transwell chambers with $8-\mu \mathrm{m}$ pore size filter membrane (Corning, USA). After $12 \mathrm{~h}$, medium was removed and cells were fixed with paraformaldehyde. The cells were washed and stained with hematoxylin-eosin. The number of cells was observed and counted under a microscope.

\section{Tube Formation Assay}

The Matrigel (Corning, USA) was added to a pre-cooled plate. Matrigel was solidified in a humidified $5 \% \mathrm{CO}_{2}$ incubator at $37^{\circ} \mathrm{C}$. Then, cells were added to the Matrigel immediately at a proper density. After $8 \mathrm{~h}$, images were visualized and photographed using a phase contrast inverted microscope.

\section{Western Blot Analysis}

Cells were lysed with an ice-cold RIPA buffer (Beyotime, China). After centrifuge, the BCA Protein Assay Reagent (Beyotime, China) was used to quantify the protein concentration of lysates. Western blot was performed in accordance with the standard protocol everywhere. SPI1 and $\beta$-actin antibodies were obtained from Cell Signal Technology (CST, USA). Membranes were subsequently incubated with HRP-conjugated secondary antibodies at room temperature. The band images were obtained from Image Lab after adding chemiluminescent substrate (Millipore, USA). Image Lab software was used to calculate relative integrated density values.

\section{Statistical Analysis}

Statistical analyses for each assay were carried out by using SPSS v20.0. Experimental results of three triplicates are represented as the mean \pm standard deviation. Statistical significance between groups was determined using Student's $t$-test or the one-way ANOVA method. A $P$-value of less than 0.05 was considered statistically significant.

\section{Results}

\section{Altered IncRNA FTX Expression in Stroke Patients Blood Samples and OGD Treated BMECs}

In order to explore the function of IncRNA FTX in IS, we firstly determined the relative expression of lncRNA FTX in stroke patients blood samples and in brain tissues of MCAO 
mice by qRT-PCR. The result displayed that lncRNA FTX was markedly decreased in stroke patients blood samples compared with healthy controls (Figure 1A). Similarly, evident reduction of IncRNA FTX in brain tissues of MCAO mice could be observed in comparison to those without MCAO treatment (Figure 1B). After OGD/R treatment for $12 \mathrm{~h}$, BMECs exhibited remarkably lower level of lncRNA FTX (Figure 1C).

\section{LncRNA FTX Overexpression Promotes Proliferation, Migration, and Angiogenesis of OGD-Treated BMECs}

After OGD-treated BMECs model establishment, we identified the effect of IncRNA FTX on cell proliferation. Followed by transfection with lncRNA FTXpcDNA3.1, BMECs were performed by CCK-8 assay. It unveiled that lncRNA FTX group displayed superior cell viability, which was compared with the pcDNA group (Figure 2A). For the purpose of further figuring out the function of IncRNA FTX on BMECs, migration and tube formation assays were executed. As shown in Figure $2 \mathrm{~B}$ and $\mathrm{C}$, it could be recognized that the cell migration ability in lncRNA FTX overexpression group was much higher than that in the pcDNA group. Besides, the ability of angiogenesis was also enhanced in the lncRNA FTX overexpression group (Figure 2D).

\section{LncRNA FTX Serves as a Competing} Endogenous RNA (ceRNA) for miR-342-3p

To identify the exact mechanism of lncRNA FTX in IS, we predicted the target of IncRNA FTX by LncBase Predicted v.2. MiR-342-3p gained the highest score in both databases (Figure 3A). We observed that IncRNA FTX contained potential binding sites for miR-342-3p (Figure 3B). Following that, the interaction between IncRNA FTX and miR342-3p was verified by dual-luciferase reporter assays. The result elucidated that relative luciferase activity was significantly reduced by co-transfection of IncRNA FTXWT and miR-342-3p mimics (Figure 3B), whereas there was no statistical change when cotransfection of IncRNA FTXmut and miR-342-3p mimics (Figure 3B).

In addition, in order to verify whether IncRNA FTX takes part in regulating expression of miR-342-3p, we overexpressed or knocked down lncRNA FTX in BMECs. The qRT-PCR showed that the level of miR342-3p was upregulated obviously followed by lncRNA FTX knockdown, while downregulated when overexpressing lncRNA FTX in BMECs (Figure 3C). Meanwhile, we detected a level of miR-342-3p between stroke patients and healthy people's blood samples. qRT-PCR indicated that miR-342-3p was upregulated in stroke patients (Figure 3D). As a result, we transfected miR-342-3p inhibitor into BMECs. CCK-8 assay implied that miR-342-3p inhibition could increase the cell viability under $\mathrm{OGD} / \mathrm{R}$ treatment (Figure 3E). It could be observed that the cell
A

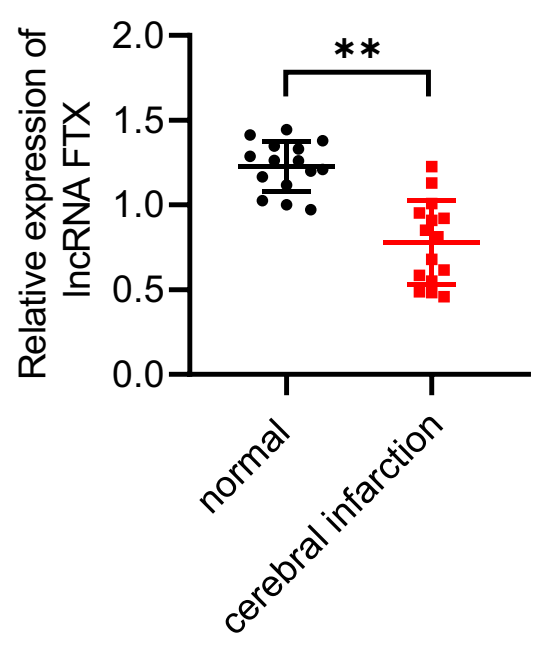

B

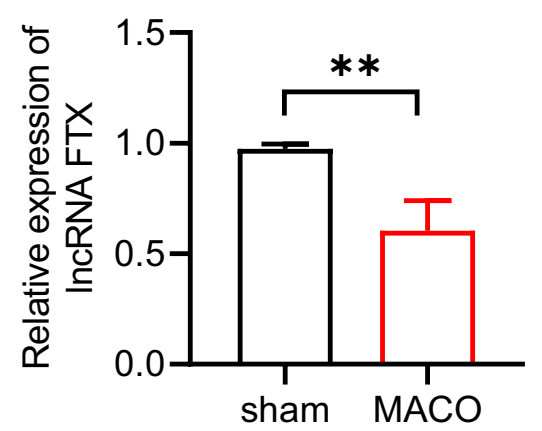

\section{C}

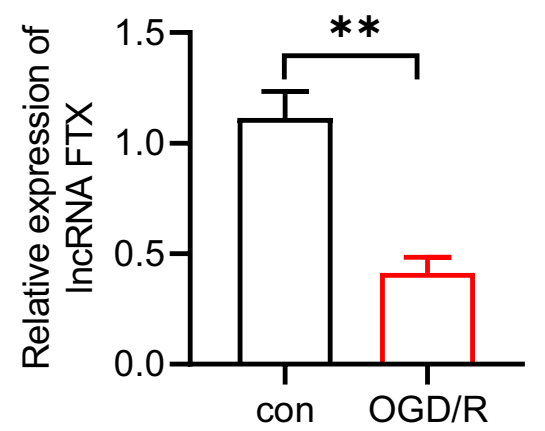

Figure I LncRNA FTX in stroke patients and OGD/R treated BMECs. (A) RTqPCR was carried out to detect the level of IncRNA FTX in blood samples obtained from stroke patients $(n=15)$ and healthy individuals. (B) Relative expression of IncRNA FTX was detected by RT-qPCR in MCAO mice brain tissues. (C) Relative expression of IncRNA FTX in oxygen and glucose deprivation/reoxygenation (OGD/R)-induced BMECs was detected by RT-qPCR. All values were represented by the mean \pm standard, $* * p<0.01$. 
A
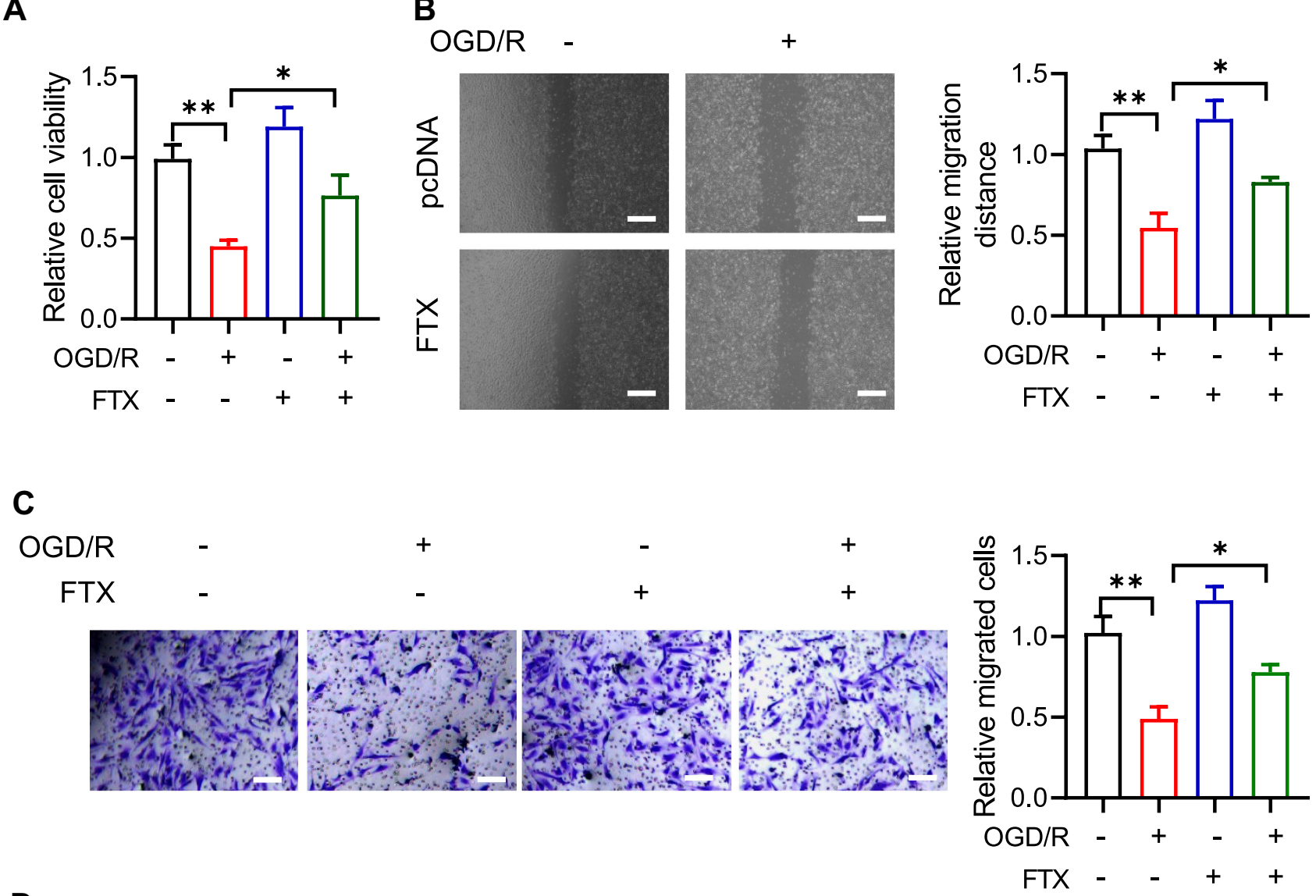

D
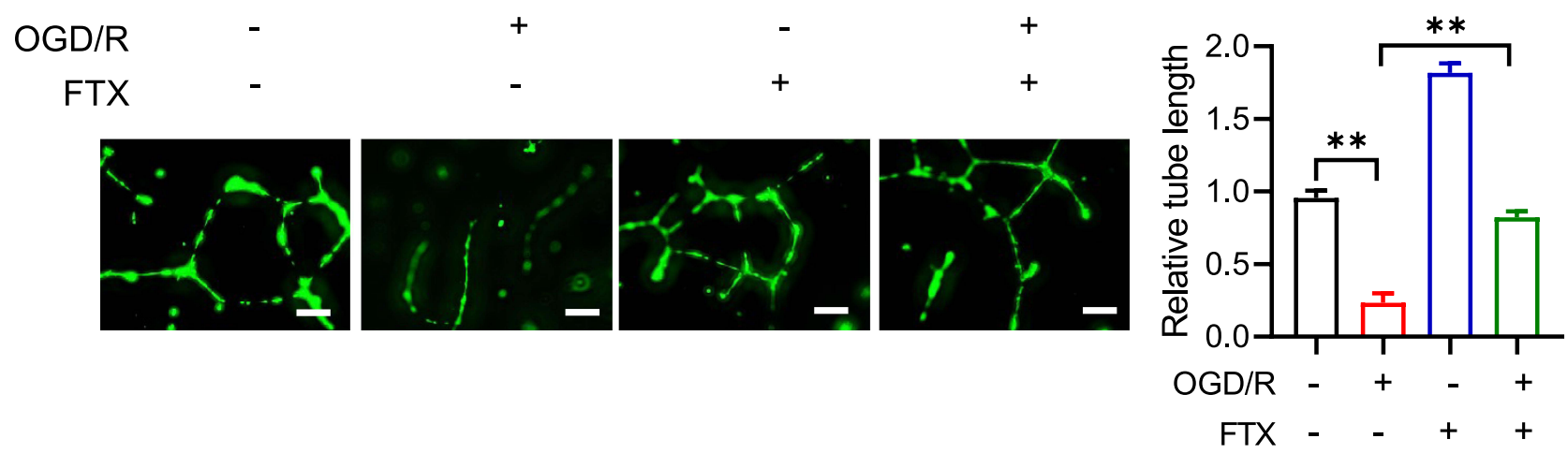

Figure 2 LncRNA FTX prevents cell proliferation, migration, angiogenesis in OGD/R treated BMECs. (A) Proliferation of BMECs was detected by CCK-8 assay. (B and C) Migration ability was measured by wound healing migration assays and transwell assays. (D) Tube formation assay was used to distinguish angiogenesis ability in BMECs. All values were represented by the mean \pm standard deviation. $*_{p}<0.05, * * p<0.01$.

migration ability was much higher in the miR-342-3p inhibitor group (Figure 3F). Moreover, angiogenesis was also promoted when inhibiting miR-342-3p in BMECs (Figure 3G).

\section{SPII is Targeted by miR-342-3p}

Subsequently, we predicted the target genes of miR-342$3 p$ and it suggested that SPI1 was a potential target. It was recognized that there were conserved binding sites between 3'UTR of SPI1 and miR-342-3p (Figure 4A). The dual-luciferase reporter assay revealed that relative luciferase activity was reduced by co-transfection of SPI1 WT and miR-342-3p mimics, which was enhanced by SPI1 mut and miR-342-3p mimics co-transfection (Figure 4A). To verify this hypothesis, the lncRNA FTX plasmids and/or miR-342-3p mimics were transfected into 
A

\begin{tabular}{|cccc|}
\hline Gene & miRNA & $\underline{\text { Score }}$ & DIANALinks \\
FTX & hsa-miR-342-3p & 0.998 & mT TB InE mP \\
\hline FTX & hsa-miR-5003-3p & 0.994 & mT TB InE mP \\
\hline FTX & hsa-miR-875-5p & 0.991 & mT TB InE mP \\
\hline FTX & hsa-miR-875-5p & 0.988 & mT TB InE mP \\
\hline FTX & hsa-miR-875-5p & 0.988 & mT TB InE mP \\
\hline FTX & hsa-miR-875-5p & 0.985 & mT TB InE mP \\
\hline FTX & hsa-miR-6823-3p & 0.982 & mT TB InE mP \\
\hline FTX & hsa-miR-6823-3p & 0.981 & mT TB InE mP \\
\hline FTX & hsa-miR-875-5p & 0.981 & mT TB InE mP \\
\hline FTX & hsa-miR-875-5p & 0.980 & mT TB InE mP \\
\hline
\end{tabular}

B

\begin{tabular}{|rr|r|r|}
\hline FTX WT 5' & UAGCAAAACACUGUGGUGUAGUGUGAGA & 3 \\
miR-342-3p 3' & CACGCUAAA GACACACUCU & $5^{\prime}$ \\
FTX MUT 5' & UAGCAAAACACACGCUAAGUACACACUCU & $3^{\prime}$ \\
\hline
\end{tabular}

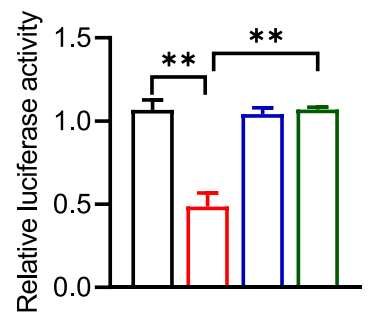

口 $W T+N C$

$\square \mathrm{WT}+\mathrm{miR}$

MUT+NC

MUT+miR
C

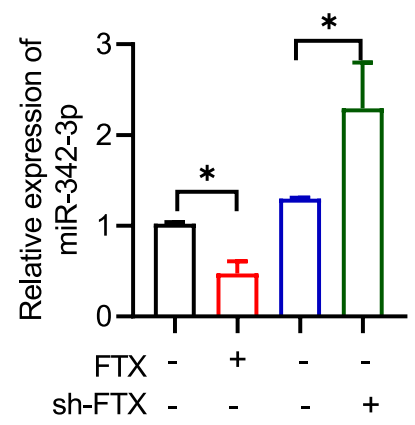

$\mathbf{F}$

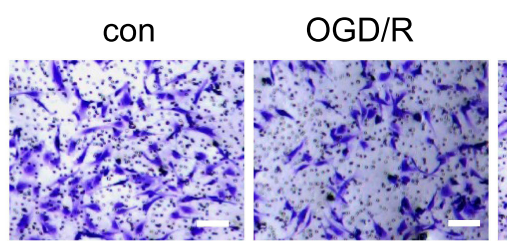

$\mathbf{G}$

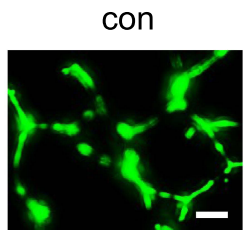

D

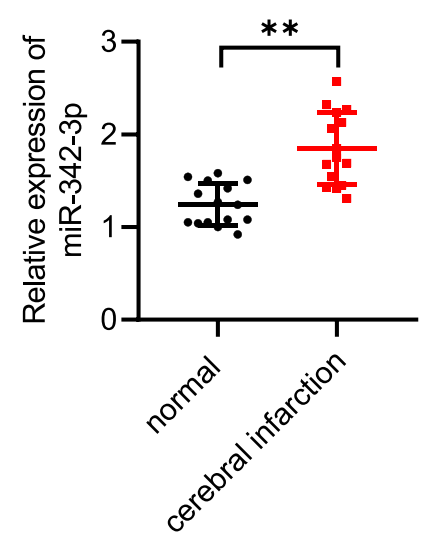

OGD/R+NC OGD/R+inhibitor

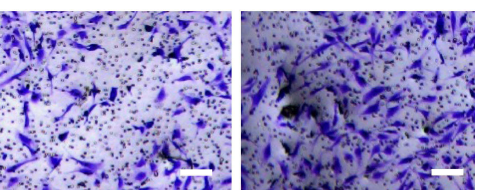

OGD/R+NC OGD/R+inhibitor
E
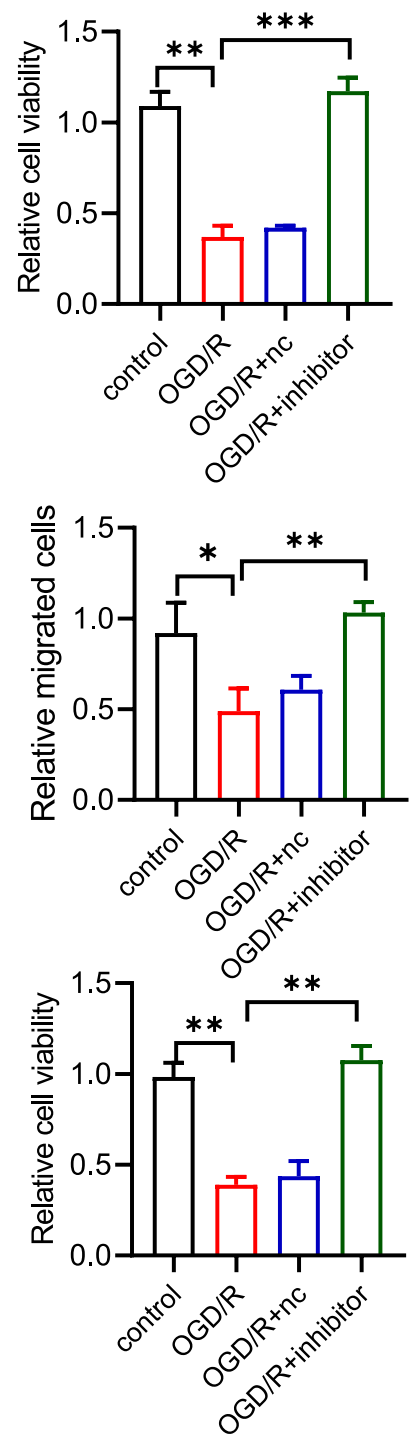

Figure 3 LncRNA FTX sponges miR-342-3p. (A) The microRNAs lists and scores on predicted by using LncBase Predicted v.2. (B) Predicted miR-342-3p binding sites in IncRNA FTX and dual luciferase report assay in WT- IncRNA FTX or MUT- IncRNA FTX co-transfected with miR-NC or miR-342-3p mimics. (C) Level of miR342-3p in BMECs transfected with shR- IncRNA FTX or oe IncRNA FTX. (D) miR342-3p expression in blood from stroke patients. (E) Proliferation of BMECs was detected by CCK8 assay. (F) Migration ability was measured by transwell assays. (G) Tube formation assay was used to distinguish angiogenesis ability in BMECs. All values were represented by the mean \pm standard deviation. ${ }^{*} p<0.05$, **p ${ }^{*}<0.01$, ${ }^{* * *} p<0.001$. 
BMECs. Western blot indicated that the IncRNA FTX overexpression appreciably augmented the expression of SPI1, while miR-342-3p mimics showed reverse trend (Figure 4B). The result of qRT-PCR showed that SPI1 was down-regulated in MCAO mice brain tissues (Figure 4C). Finally, tube formation assay demonstrated that the elevated level of angiogenesis after lncRNA FTX overexpression was neutralized by miR-342-3p overexpression and SPI1knockdown (Figure 4D).

\section{Discussion}

Abnormal expression of lncRNAs is strictly related to stroke. ${ }^{22}$ In our study, we confirmed that lncRNA FTX expression was downregulated in OGD-induced BMECs. Additionally,
A

SPI1 WT 5' GUUGCAUGUGUGCGAGUGGCUGUGUGAGU 3'

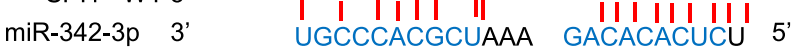
SPI1 MUT 5' GUUGCUGCCCACGCUGUGGGACACACUCU 3'

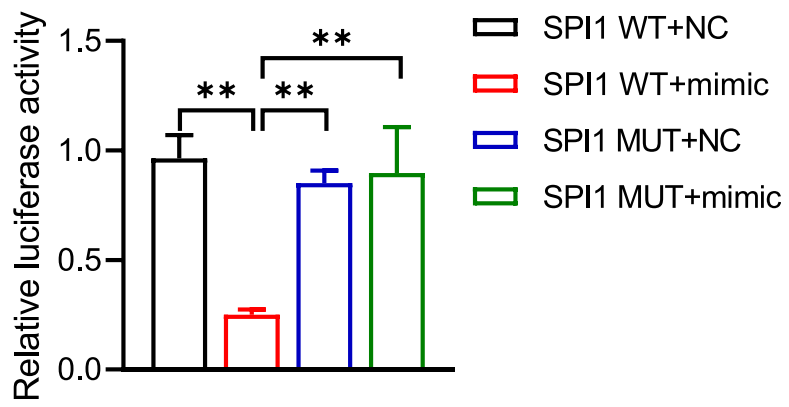

B
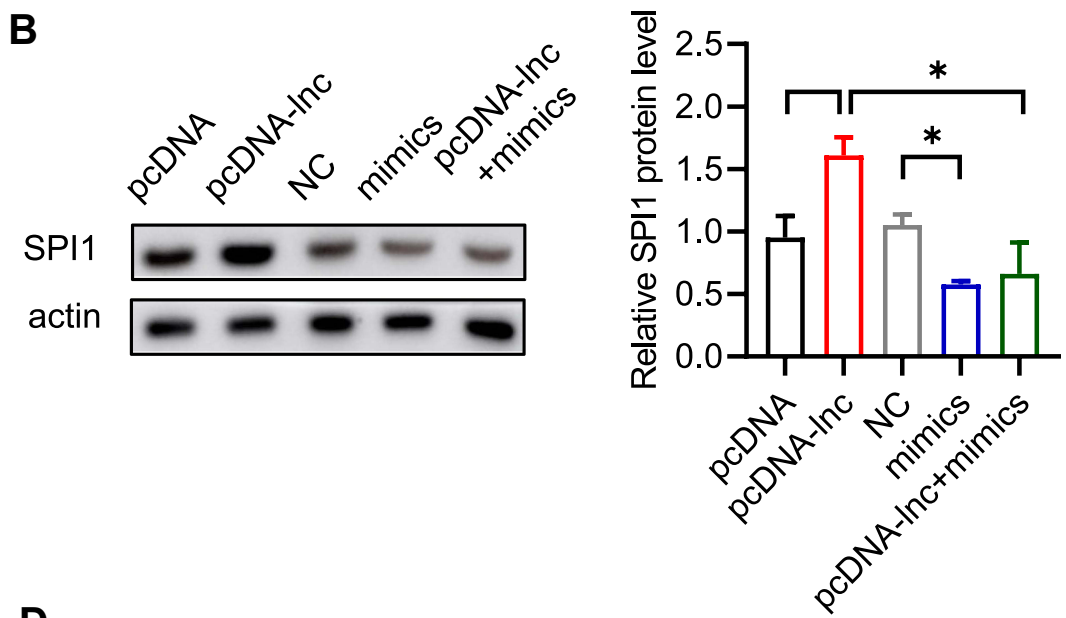

C

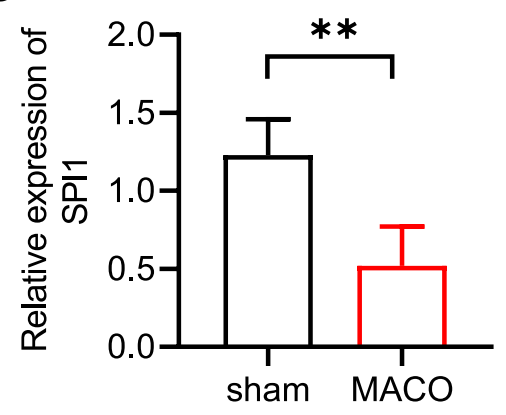

D con

OGD/R
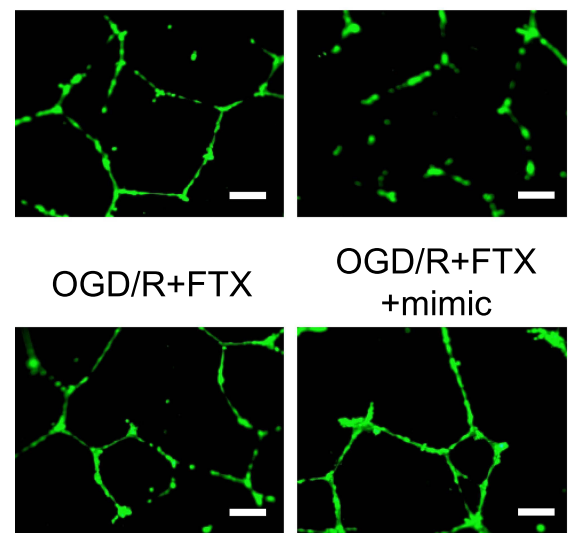

OGD/R+FTX

+ mimic

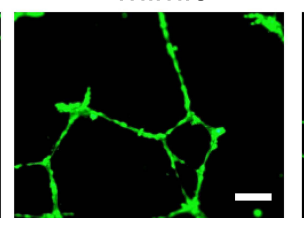

\section{OGD/R+pcDNA}
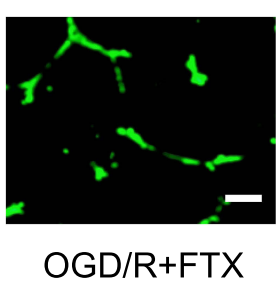

+sh-SPI1

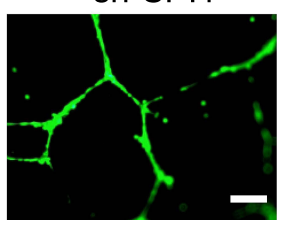

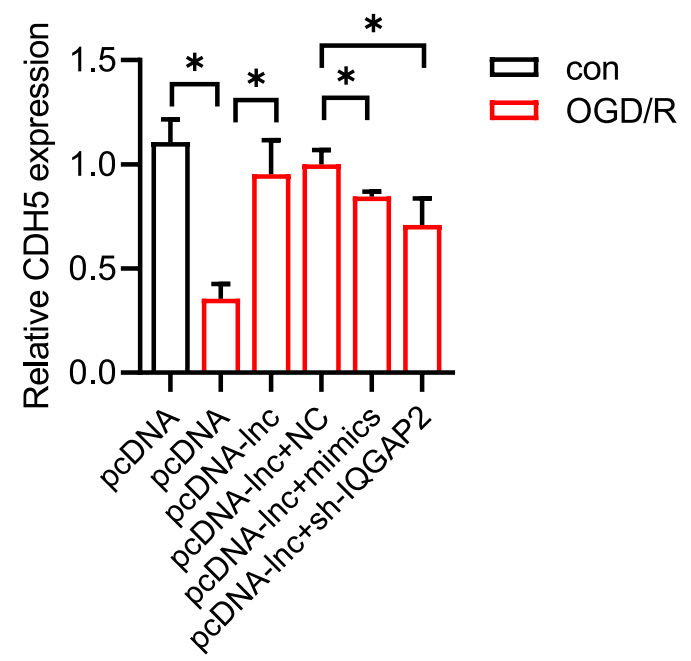

Figure 4 LncRNA FTX promotes angiogenesis through regulating miR-342-3p /SPII axis. (A) Binding sites motif between miR-342-3P and SPII and dual luciferase assay in WT- SPII or MUT- SPII BMECs co-transfected with miR-NC or miR-3423p mimics. (B) The protein SPII level was detected by WB. (C) qRT-PCR showed that SPII mRNA level in MCAO mice brain tissues. (D) Tube formation assay was used to detect the ability of angiogenesis in HRECs. All values were represented by the mean \pm standard deviation. ${ }^{*} p<0.05$, ${ }^{* *} p<0.01$. 
IncRNA FTX promoted proliferation and angiogenesis through miR-342-3p/SPI1 axis in OGD-induced BMECs, which may be an innovative target for ischemic stroke treatment.

Ischemic stroke could result in BMECs death and blood-brain barrier disruption, leading to neurological injury. ${ }^{23}$ Angiogenesis formed by BMECs could enhance the survival rate of stroke patients. ${ }^{24}$ LncRNA FTX has been reported to be related to many cancers and has promoted the proliferation of cancer cells. ${ }^{20}$ LncRNA FTX has known functions related to vasculogenic mimicry formation and tumorigenesis in osteosarcoma. ${ }^{21}$ In the present study, we indicated that IncRNA FTX promoted cell viability, cell migration, and angiogenesis. We further investigated the mechanism of the lncRNA FTX function. LncRNA has been indicated to be a sponge for miRNAs. We verified that IncRNA FTX could interact with miR342-3p. MiR-342-3p in endothelial cells was compromised in T2DM, and inhibition of miR-342-3p could block vasculogenesis by decreasing proliferation and migration of endothelial cell. ${ }^{25}$ In line with the findings, we found that IncRNA FTX directly bound with miR-342-3p in BMECs. MiR-342-3p inhibition could promote proliferation and lumen formation. Additionally, lncRNA FTX overexpression enhanced SPI1 expression. SPI1/PU.1 could bind to a purine-rich sequence, regulating genes expression, which is known as a transcriptional activator. ${ }^{26-28}$ We found that the 3'-UTR of SPI1 directly bound with miR-342-3p in BMECs. Herein, we also found that the upregulated angiogenesis after lncRNA FTX overexpression was outweighed by miR-342-3p overexpression and SPI1 knockdown.

In conclusion, it was indicated that lncRNA FTX enhanced the migration and angiogenesis in OGD-treated BMECs through the miR-342-3p/SPI1 axis, providing an exact mechanism of lncRNA FTX and miR-342-3p in stroke. Our findings indicated that overexpression of lncRNA FTX and knockdown of miR-342-3p showed a protective role in stroke. Therefore, lncRNA FTX and miR-342-3p could be used as a therapeutic target for the treatment of stroke in the future.

\section{Conclusions}

In summary, lncRNA FTX enhanced the angiogenesis in stroke by acting as a sponge of miR-342-3p to regulate the expression of SPI1 level. Our findings indicated that overexpression of lncRNA FTX and knockdown of miR-342-
$3 \mathrm{p}$ showed a protective role in stroke, which could provide the new targets for stroke treatment.

\section{Data Sharing Statement}

All data generated or analyzed during this study are included in this published article.

\section{Ethics Approval}

The study received approval from the Affiliated Huaian No.1 People's Hospital of Nanjing Medical University (YX-2021-071-01). All participants were informed about the purpose of the study, and that it was conducted in accordance with the Declaration of Helsinki.

\section{Acknowledgments}

We would like to express our gratitude to all those who financed the subject.

\section{Funding}

Sponsors had no such involvement.

\section{Disclosure}

The authors declare that they have no competing interests.

\section{References}

1. Owolabi MO, Thrift AG, Martins S, et al. The state of stroke services across the globe: report of World Stroke Organization-World Health Organization surveys. Int $J$ Stroke. 2021:17474930211019568. doi:10.1177/174749930211019568

2. Weaver NA, Kuijf HJ, Aben HP, et al. Strategic infarct locations for post-stroke cognitive impairment: a pooled analysis of individual patient data from 12 acute ischaemic stroke cohorts. Research Support, Non-U.S. Gov't. Lancet Neurol. 2021;20(6):448-459. doi:10.1016/S1474-4422(21)00060-0

3. Li L, Poon MTC, Samarasekera NE, et al. Risks of recurrent stroke and all serious vascular events after spontaneous intracerebral haemorrhage: pooled analyses of two population-based studies. Research Support, Non-U.S. Gov't. Lancet Neurol. 2021;20(6):437-447. doi:10.1016/S1474-4422(21)00075-2

4. Zhang Y, Hua W, Li Z, et al. Efficacy and safety of a novel thrombectomy device in patients with acute ischemic stroke: a randomized controlled trial. Case Reports. Front Neurol. 2021;12:686253. doi:10.3389/fneur.2021.686253

5. Rothwell PM, Buchan AM. A new thrombolytic drug for acute ischaemic stroke. Comment. Lancet Neurol. 2021;20(9):687-689. doi:10.1016/S1474-4422(21)00256-8

6. Potente M, Gerhardt H, Carmeliet P. Basic and therapeutic aspects of angiogenesis. Research Support, Non-U.S. Gov't Review. Cell. 2011;146(6):873-887. doi:10.1016/j.cell.2011.08.039

7. Marti HJ, Bernaudin M, Bellail A, et al. Hypoxia-induced vascular endothelial growth factor expression precedes neovascularization after cerebral ischemia. Research Support, Non-U.S. Gov't. Am J Pathol. 2000;156(3):965-976. doi:10.1016/S0002-9440(10)64964-4

8. Krupinski J, Kaluza J, Kumar P, Kumar S, Wang JM. Role of angiogenesis in patients with cerebral ischemic stroke. Stroke. 1994;25 (9):1794-1798. doi:10.1161/01.str.25.9.1794 
9. Pan JR, Li Y, Pei Z, Li XP, Peng Y, Wang YD. Hypoxic tissues are associated with microvessel density following brain ischemiareperfusion. Research Support, Non-U.S. Gov't. Neurol Sci. 2010;31(6):765-771. doi:10.1007/s10072-010-0441-z

10. Gregorius J, Wang C, Stambouli O, et al. Small extracellular vesicles obtained from hypoxic mesenchymal stromal cells have unique characteristics that promote cerebral angiogenesis, brain remodeling and neurological recovery after focal cerebral ischemia in mice. Research Support, Non-U.S. Gov't. Basic Res Cardiol. 2021;116(1):40. doi:10.1007/s00395-021-00881-9

11. Jin Q, Cheng J, Liu Y, et al. Improvement of functional recovery by chronic metformin treatment is associated with enhanced alternative activation of microglia/macrophages and increased angiogenesis and neurogenesis following experimental stroke. Research Support, Non-U.S Gov't. Brain Behav Immun. 2014;40:131-142. doi:10.1016/j. bbi.2014.03.003

12. Manoonkitiwongsa PS, Jackson-Friedman C, McMillan PJ, Schultz RL, Lyden PD. Angiogenesis after stroke is correlated with increased numbers of macrophages: the clean-up hypothesis. Review. J Cereb Blood Flow Metab. 2001;21(10):1223-1231. doi:10.1097/ 00004647-200110000-00011

13. Wilusz JE, Sunwoo H, Spector DL. Long noncoding RNAs: functional surprises from the RNA world. Research Support, N.I.H., Extramural Research Support, Non-U.S. Gov't Review. Genes Dev. 2009;23(13):1494-1504. doi:10.1101/gad.1800909

14. Mercer TR, Mattick JS. Structure and function of long noncoding RNAs in epigenetic regulation. Research Support, Non-U.S. Gov't Review. Nat Struct Mol Biol. 2013;20(3):300-307. doi:10.1038/ nsmb. 2480

15. Masutani S, Sasaki Y, Imaoka S, et al. [Changes of serum interleukin 6 (IL6) and acute phase protein after hepatectomy in patients with chronic liver disease]. Nihon Geka Gakkai Zasshi. 1993;94 (10):1071-1077. Japanese.

16. Bao MH, Szeto V, Yang BB, Zhu SZ, Sun HS, Feng ZP. Long noncoding RNAs in ischemic stroke. Research Support, Non-U.S. Gov't Review. Cell Death Dis. 2018;9(3):281. doi:10.1038/s41419-0180282-X

17. Zhang B, Wang D, Ji TF, Shi L, Yu JL. Overexpression of IncRNA ANRIL upregulates VEGF expression and promotes angiogenesis of diabetes mellitus combined with cerebral infarction by activating NF-kappaB signaling pathway in a rat model. Oncotarget. 2017;8 (10):17347-17359. doi:10.18632/oncotarget.14468

18. Zhang L, Luo X, Chen F, et al. LncRNA SNHG1 regulates cerebrovascular pathologies as a competing endogenous RNA through HIF-1alpha/ VEGF signaling in ischemic stroke. Research Support, Non-U.S. Gov't. J Cell Biochem. 2018;119(7):5460-5472. doi:10.1002/jcb.26705
19. Deng W, Fan C, Shen R, Wu Y, Du R, Teng J. Long noncoding MIAT acting as a ceRNA to sponge microRNA-204-5p to participate in cerebral microvascular endothelial cell injury after cerebral ischemia through regulating HMGB1. J Cell Physiol. 2020;235(5):4571-4586. doi:10.1002/jcp.29334

20. Lin Y, Shen Y, Chen J, Hu C, Zhou Z, Yuan C. The function of LncRNA FTX in several common cancers. Research Support, NonU.S. Gov't. Curr Pharm Des. 2021;27(20):2381-2386. doi:10.2174/ 1381612826666201029164036

21. Ren K, Ni Y, Li X, et al. Expression profiling of long noncoding RNAs associated with vasculogenic mimicry in osteosarcoma. Research Support, Non-U.S. Gov't. J Cell Biochem. 2019;120 (8):12473-12488. doi:10.1002/jcb.28514

22. Vasudeva K, Dutta A, Munshi A. Role of lncRNAs in the development of ischemic stroke and their therapeutic potential. Review. Mol Neurobiol. 2021;58(8):3712-3728. doi:10.1007/s12035-021-02359-0

23. Nian K, Harding IC, Herman IM, Ebong EE. Blood-brain barrier damage in ischemic stroke and its regulation by endothelial mechanotransduction. Review. Front Physiol. 2020;11:605398. doi:10.3389/ fphys.2020.605398

24. Annex BH, Cooke JP. New directions in therapeutic angiogenesis and arteriogenesis in peripheral arterial disease. Circ Res. 2021;128 (12):1944-1957. doi:10.1161/CIRCRESAHA.121.318266

25. Cheng S, Cui Y, Fan L, Mu X, Hua Y. T2DM inhibition of endothelial miR-3423p facilitates angiogenic dysfunction via repression of FGF11 signaling. Biochem Biophys Res Commun. 2018;503 (1):71-78. doi:10.1016/j.bbrc.2018.05.179

26. Zimmer TS, Korotkov A, Zwakenberg S, et al. Upregulation of the pathogenic transcription factor SPI/PU.1 in tuberous sclerosis complex and focal cortical dysplasia by oxidative stress. Brain Pathol. 2021;31(5):e12949. doi:10.1111/bpa.12949

27. Wheat JC, Sella Y, Willcockson M, et al. Single-molecule imaging of transcription dynamics in somatic stem cells. Research Support, NonU.S. Gov’t. Nature. 2020;583(7816):431-436. doi:10.1038/s41586020-2432-4

28. Sun J, Chen J, Li T, et al. ROS production and mitochondrial dysfunction driven by PU.1-regulated NOX4-p22(phox) activation in Abeta-induced retinal pigment epithelial cell injury. Observational Study Research Support, Non-U.S. Gov't. Theranostics. 2020;10(25):11637-11655. doi:10.7150/thno.48064
Neuropsychiatric Disease and Treatment

\section{Publish your work in this journal}

Neuropsychiatric Disease and Treatment is an international, peerreviewed journal of clinical therapeutics and pharmacology focusing on concise rapid reporting of clinical or pre-clinical studies on a range of neuropsychiatric and neurological disorders. This journal is indexed on PubMed Central, the 'PsycINFO' database and CAS, and is the official journal of The International Neuropsychiatric Association (INA). The manuscript management system is completely online and includes a very quick and fair peer-review system, which is all easy to use. Visit http://www.dovepress.com/testimonials.php to read real quotes from published authors. 